\title{
Erratum to: Toxicity of nanocrystal quantum dots: the relevance of surface modifications
}

\author{
Akiyoshi Hoshino $\cdot$ Sanshiro Hanada \\ Kenji Yamamoto
}

Received: 4 November 2010/Accepted: 24 February 2011/Published online: 17 April 2011

(C) Springer-Verlag 2011

\section{Erratum to: Arch Toxicol}

DOI 10.1007/204-011-0695-0

Unfortunately, there has been a mistake concerning the corresponding author's e-mail. It should be written correctly as follows:

Akiyoshi Hoshino
e-mail: hoshinoa@nih.go.jp

The online version of the original article can be found under doi:10.1007/s00204-011-0695-0.

\footnotetext{
A. Hoshino $(\bowtie) \cdot S$. Hanada $\cdot$ K. Yamamoto Vice Director's Lab, Research Institute,

National Center for Global Health and Medicine,

Toyama 1-21-1, Shinjuku-ku, Tokyo 162-8655, Japan

e-mail: hoshinoa@nih.go.jp
} 